\title{
Suppression of CLEC3A inhibits osteosarcoma cell proliferation and promotes their chemosensitivity through the AKT1/mTOR/HIF1 $\alpha$ signaling pathway
}

\author{
CHONG REN $^{1 *}$, RUNSANG PAN $^{1 *}$, LISONG HOU ${ }^{1}$, HUAPING WU $^{1}$, JUNKANG SUN $^{1}$, \\ WENGUANG ZHANG $^{1}$, XIAOBIN TIAN ${ }^{2}$ and HOUPING CHEN ${ }^{1}$ \\ ${ }^{1}$ Department of Orthopedics, Guiyang Maternal and Child Health-Care Hospital; \\ ${ }^{2}$ Department of Orthopedics, Clinical Medical College of Guizhou Medical University, \\ Guiyang, Guizhou 550000, P.R. China
}

Received June 15, 2019; Accepted December 6, 2019

DOI: $10.3892 / \mathrm{mmr} .2020 .10986$

\begin{abstract}
Osteosarcoma (OS) is a primary malignant tumor that occurs in bone, and mainly affects children and adolescents. C-type lectin domain family 3 member A (CLEC3A) is a member of the C-type lectin superfamily, which regulates various biological functions of cells. The present study aimed to identify the effects and related mechanisms of CLEC3A in the proliferation and chemosensitivity of OS cells. The expression of CLEC3A in OS was analyzed using the Gene Expression Omnibus data profile GSE99671, and its expression in OS samples was verified using reverse transcription-quantitative PCR (RT-qPCR) and immunohistochemical staining. The relationship between the expression of CLEC3A and clinical traits in patients with OS was also analyzed, including age, tumor size, TNM stage and lymph node metastasis. Cell Counting Kit- 8 assays, colony formation assays and cell cycle distribution analysis were used to determine the roles of CLEC3A in the proliferation and chemosensitivity of OS cells. Finally, RT-qPCR and western blotting were used to demonstrate the relationship between CLEC3A and the AKT1/mTOR/hypoxia-inducible factor 1- $\alpha$ (HIF1 $\alpha$ ) pathway. Both the mRNA and protein expression levels of CLEC3A were increased in OS tissues compared with adjacent
\end{abstract}

Correspondence to: Dr Houping Chen, Department of Orthopedics, Guiyang Maternal and Child Health-Care Hospital, 63 Ruijin Road, Guiyang, Guizhou 550000, P.R. China

E-mail: chenhouping@126.com

Dr Xiaobin Tian, Department of Orthopedics, Clinical Medical College of Guizhou Medical University, 9 Beijing Road, Guiyang, Guizhou 550000, P.R. China

E-mail: txb6@vip.163.com

*Contributed equally

Key words: osteosarcoma, c-type lectins, proliferation, chemosensitivity non-tumor tissues, and this was positively associated with TNM stage and lymph node metastasis. The genetic knockdown of CLEC3A with small interfering RNA decreased OS cell proliferation and colony formation, and induced $\mathrm{G} 1$ phase arrest, whereas the overexpression of CLEC3A increased OS cell proliferation and colony formation, and alleviated G1 phase arrest. The suppression of CLEC3A also promoted enhanced the chemosensitivity of OS cells to doxorubicin (DOX) and cisplatin (CDDP); it also inhibited the expression of AKT1, mTOR and HIF1 $\alpha$, further to the nuclear localization of HIF1 $\alpha$, and HIF1 $\alpha$ target gene expression levels, including VEGF, GLUT1 and MCL1 were also decreased. Furthermore, treatment with the AKT activator SC79 blocked the inhibitory effects of CLEC3A silencing in OS cells. In conclusion, these findings suggested that CLEC3A may function as an oncogene in OS, and that the suppression of CLEC3A may inhibit OS cell proliferation and promote chemosensitivity through the AKT1/mTOR/HIF1 $\alpha$ signaling pathway.

\section{Introduction}

Osteosarcoma (OS) is one of the most common primary malignancies that occurs in bone tissues, resulting in $~ 9 \%$ of cancer-related deaths in adolescents and children between the ages of 10 and 24 (1); in the past 60 years, a second peak of incidence has been observed (2). OS demonstrates a high probability to metastasize and damage surrounding tissues, especially within the lung; however, despite the existence of surgical excision and the development of neoadjuvant chemotherapies for patients with OS over the past few decades, the prognosis of OS for 20 year survival remains $<20 \%$ (3). This poor prognosis is largely due to the anatomical location, tumor size, tumor stage, presence or absence of local recurrence and metastasis, in addition to ineffective chemotherapy regimens (4). Thus, the development of new patient treatment strategies for OS is urgently required. There is evidence to suggest that the regulation of oncogenes and tumor suppressors serve vital roles in the progression of OS (5). Hence, it is of great importance to identify the molecular mechanism of OS metastasis to improve the diagnosis and treatment of osteosarcoma. 
The C-type lectins are the largest family of lectins and belong to a group of proteins involved in various functions, such as cell differentiation, migration and proliferation. Most members of the family have the C-type carbohydrate recognition domain located on the outer surface of the cell, which under physical stress, can specifically identify and bind to proteins, lipids and carbohydrates in a $\mathrm{Ca}^{2+}$-dependent manner (6). Dysfunctional C-type lectins have been reported in various pathological states, including cancer; for example, Wang et al (7) observed that C-type-lectin-like-2 promoted the proliferation and migration of gastric cancer cells by regulating the AKT signaling pathway. Ni et al (8) demonstrated that high expression levels of the C-type lectin domain family 3 member A (CLEC3A) were positively associated with poor prognosis in patients with invasive ductal carcinoma of the breast. In addition, as a member of the C-type lectin superfamily, CLEC3A was originally identified in cartilage, and a previous study reported that CLEC3A was involved in bone formation (6); however, the effect and molecular mechanism of CLEC3A in OS is largely unknown.

In the present study, the expression levels of CLEC3A were observed to be increased in OS tissues, which was associated with TNM stage and lymph node metastasis. Furthermore, the suppression of CLEC3A using small interfering RNA (siRNA) inhibited OS cell proliferation and promoted their chemosensitivity through the AKT1/mTOR/hypoxia-inducible factor 1- $\alpha$ (HIF1 $\alpha$ ) signaling pathway. These findings may contribute to the development of a novel targeted therapy for the diagnosis and treatment of OS.

\section{Materials and methods}

Patient studies. The present study was approved by the ethics committee of the Maternal and Child Health Hospital of Guiyang Province and was performed in accordance with the principles embodied in the Declaration of Helsinki. Written informed consent was obtained from all patients who provided samples. Clinical OS tissue and tumor-adjacent normal samples from patients with OS were obtained from the Maternal and Child Health Hospital of Guiyang Province between June 2015 and March 2019. A total of 30 patients (male/female=17/13; age range: 8-66 years; mean age: 17.3) enrolled in the present study: All of them provided OS tissues and 15 of them also provided adjacent tissues.

Bioinformatics method. Gene expression profile data GSE99671 was obtained from the Gene Expression Omnibus (http://www.ncbi.nlm.nih.gov/geo). This profile was provided by Ho et al (9), and includes 18 OS tissues and 18 corresponding tissues. The gene expression profile data were normalized and differently expressed analysis performed using R software (version 3.5.2; The R Foundation; https://www.r-project.org/) (10). Log fold change (LogFC) $>2$ and adjusted P-value $<0.05$ were selected as cut-offs for differentially expression.

Cell culture and reagents. The human OS cell lines SaOS-2 and MG63 were purchased from the American Type Culture Collection. Cells were cultured in DMEM (Gibco; Thermo Fisher Scientific, Inc.), supplemented with 10\% FBS
(Gibco; Thermo Fisher Scientific, Inc.) and maintained in a humidified at atmosphere at $37^{\circ} \mathrm{C}$ and $5 \% \mathrm{CO}_{2}$.

Reverse transcription-quantitative PCR (RT-qPCR). Total RNA was extracted from OS tissues $(0.5 \times 0.5 \times 0.3 \mathrm{~cm})$, adjacent tissues $(0.5 \times 0.5 \times 0.3 \mathrm{~cm})$ and cells lines (MG63 and SaSO-2, $5 \times 10^{5}$ cells ) using TRIzol ${ }^{\circledR}$ reagent [Yeasen Biotechnology (Shanghai) Co., Ltd.], according to the manufacturer's protocol. Total RNA was reverse transcribed into cDNA using the PrimeScript RT reagent kit [Yeasen Biotechnology (Shanghai) Co., Ltd.] according to the manufacturer's protocol. qPCR was subsequently performed using SYBR Green qPCR Master Mix [Yeasen Biotechnology (Shanghai) Co., Ltd.]. The following primer pairs were used for the qPCR: CLEC3A forward, 5'-CGAGGCACTAAAGTTCACAAGA-3' and reverse, 5'-CGGAGTTCCTGGGGATAACCA-3'; AKT1 forward, 5'-AGCGACGTGGCTATTGTGAAG-3' and reverse, 5'-GCCATCATTCTTGAGGAGGAAGT-3'; MCL1 forward, 5'-TGCTTCGGAAACTGGACATCA-3' and reverse, 5'-TAG CCACAAAGGCACCAAAAG-3'; GLUT1 forward, 5'-GGC CAAGAGTGTGCTAAAGAA-3' and reverse, 5'-ACAGCG TTGATGCCAGACAG-3'; VEGF forward, 5'-AGGGCAGAA TCATCACGAAGT-3' and reverse, 5'-AGGGTCTCGATT GGATGGCA-3'; $\beta$-actin forward, 5'-CATGTACGTTGCTAT CCAGGC-3' and reverse, 5'-CTCCTTAATGTCACGCAC GAT-3'. The following thermocycling conditions were used for qPCR: Initial denaturation at $95^{\circ} \mathrm{C}$ for $30 \mathrm{sec}$; and 40 cycles of $95^{\circ} \mathrm{C}$ for $30 \mathrm{sec}$ and $60^{\circ} \mathrm{C}$ for $30 \mathrm{sec}$. Expression levels were quantified using the $2^{-\Delta \Delta \mathrm{Cq}}$ method (11) and the internal reference gene $\beta$-actin acted as the control.

Immunohistochemical (IHC) staining. OS tissues were fixed using $4 \%$ paraformaldehyde for $30 \mathrm{~min}$ under room temperature, dehydrated using a Rapid Tissue Processor (Sakura Seiki Co., Ltd.) under room temperature, embedded in paraffin (Wuhan Servicobio Technology Co., Ltd.) and subsequently cut into $2-\mu \mathrm{m}$ sections. Sections were deparaffinized, and then rehydrated with xylene and a descending alcohol series at room temperature, respectively. Following restoration by sodium citrate $(100 \mathrm{mM})$ at room temperature, the sections were blocked with $3 \% \mathrm{H}_{2} \mathrm{O}_{2}$ and $5 \%$ BSA (Wuhan Servicobio Technology Co., Ltd.) at room temperature and subsequently incubated with an anti-CLEC3A primary antibody (1:400; cat. no. ab185282; Abcam) for $16 \mathrm{~h}$ at $4^{\circ} \mathrm{C}$. Following the primary incubation, the sections were incubated with a horseradish peroxidase (HRP)-conjugated secondary antibody (1:400, cat no. G1210-2-A-100, Wuhan Servicobio Technology Co., Ltd.) for $2 \mathrm{~h}$ at room temperature. The slides were subsequently stained using a Cell and Tissue Staining HRP-3,3'-diaminobenzidine kit (Wuhan Servicobio Technology Co., Ltd.) and the nuclear counterstain was used $0.2 \%$ hematoxylin at room temperature for $1 \mathrm{~min}$. The staining was visualized using an light microscope (magnification, $\mathrm{x} 100)$.

Cell transfection. CLEC3A siRNA (si-CLEC3A) and control siRNA [si-negative control (NC)] were obtained from Shanghai GenePharma, Co., Ltd.; CLEC3A overexpression plasmid and empty plasmid were purchased from Sangon Biotech Co., Ltd. The si-CLEC3A sequence was 5'-CAGAAGTCAATG 
CCTTGAAGGAAAT-3'. The si-NC sequence was 5'-ACG AGACACGAACGGAGAATT-3'. A total of 1x10 ${ }^{5}$ MG63 and SaOS-2 cells were plated into 6-well plates and upon reaching 50-60\% confluence, si-CLEC3A, control siRNA, the CLEC3A overexpression plasmid and the empty plasmid (all $10 \mu \mathrm{m}$ ) were transfected into OS cells using Lipofectamine ${ }^{\circledR} 2000$ reagent (Invitrogen; Thermo Fisher Scientific, Inc.), according to the manufacturer's protocol. Following $48 \mathrm{~h}$ of transfection, the efficiency of si-CLEC3A or CLEC3A overexpression plasmid was detected by RT-qPCR and western blotting. Similarly, subsequent experimentation was also performed after $48 \mathrm{~h}$ of transfection.

Cell viability assay. A total of $4 \times 10^{3}$ MG63 and SaOS-2 cells/well were plated into 96-well plates and co-cultured with cisplatin (CDDP, MedChemExpress LLC; 0, 0.25, 1, 2, 4 and $8 \mu \mathrm{M}$ ), doxorubicin (DOX, MedChemExpress LLC; 0, 0.25, 1, 2, 4 and $8 \mu \mathrm{M}$ ) or SC79 (MedChemExpress LLC, $5 \mu \mathrm{M}$ ) for $48 \mathrm{~h}$ at $37^{\circ} \mathrm{C}$. Subsequently, $10 \mu \mathrm{l}$ Cell Counting Kit-8 reagent (Dojindo Molecular Technologies, Inc.) was added to each well according to the manufacturer's protocol. Following incubation for $2 \mathrm{~h}$ at $37^{\circ} \mathrm{C}$, the absorbance was measured at $450 \mathrm{~nm}$ using a microplate reader. To calculate the $\mathrm{IC}_{50}$, the inhibitory rate of each concentration were exported into GraphPad Prism version 6.0 software (GraphPad Software, Inc.) and linear regression analysis performed. After obtaining the corresponding linear regression equation, inhibitory rate $=50$ was substituted into corresponding equation and the $\mathrm{IC}_{50}$ was calculated.

Colony formation assay. Following transfection for $24 \mathrm{~h}$, a total of $1 \times 10^{3}$ MG63 and SaOS-2 cells/well were plated into six-well plates. To detect chemosensitivity, CDDP $(2 \mu \mathrm{M})$, DOX $(2 \mu \mathrm{M})$ or SC79 $(5 \mu \mathrm{M})$ were added in the well on the second day for 2 weeks at $37^{\circ} \mathrm{C}$. The medium was changed every third day. At 2 weeks after plating, the cells were fixed with $4 \%$ paraformaldehyde for $30 \mathrm{~min}$ at room temperature and subsequently stained with $0.1 \%$ crystal violet for $15 \mathrm{~min}$ at room temperature. Colonies with area $>10 \mathrm{~mm}^{2}$ were counted.

Cell cycle analysis. Following transfection for $24 \mathrm{~h}, 1 \times 10^{6}$ MG63 and SaOS-2 cells were harvested by centrifugation $(600 \mathrm{x} \mathrm{g})$ at room temperature for $5 \mathrm{~min}$ and fixed with PBS containing $75 \%$ ice-cold ethanol overnight at $-20^{\circ} \mathrm{C}$. Cells were subsequently incubated with $10 \mu \mathrm{g} / \mathrm{ml}$ propidium iodide solution (Invitrogen; Thermo Fisher Scientific, Inc.) at room temperature. Flow cytometry was performed using a BD FACSCalibur ${ }^{\mathrm{TM}}$ flow cytometer (BD Biosciences; version: 343020 ) to detect the cell cycle distribution and FlowJo (version no. 7.6.1; FlowJo LLC) was used for analysis .

Western blotting. Total protein of $2 \times 10^{6} \mathrm{MG} 63$ and SaOS-2 cells was extracted using RIPA lysis buffer (Boster Biological Technology) containing 1:50 EDTA-free Protease Inhibitor Cocktail (Boster Biological Technology) and phenylmethylsulfonyl fluoride (Boster Biological Technology). Cell lysates were centrifuged $(8,000 \mathrm{x} \mathrm{g})$ for $15 \mathrm{~min}$ at $4^{\circ} \mathrm{C}$ to separate protein from cellular debris. Total protein was quantified using a Bicinchoninic Acid assay (Boster Biological Technology) and proteins $(30 \mu \mathrm{g})$ were separated by $10 \%$ SDS-PAGE.
The separated proteins were subsequently transferred onto PVDF membranes (Merck KGaA) and blocked in 5\% skim milk with TBS-Tween-20 (0.1\%) for $2 \mathrm{~h}$ (TBST) at room temperature. The membranes were incubated with the following primary antibodies at $4^{\circ} \mathrm{C}$ overnight: Anti-CLEC3A (1:1,000; cat no. H00010143-B01; Abnova), anti-AKT (1:1,000; cat no. 10176-2-AP), anti-mTOR (1:1,000; cat no. 66888-1-Ig), anti-HIF1 $\alpha$ (1:1,000; cat no. 20960-1-AP), anti-GLUT1 (1:1,000; cat no. 21829-1-AP,), anti-VEGF (1:1,000; cat no. 19003-1-AP), anti-MCL1 (1:1,000; cat no. 16225-1-AP) and anti- $\beta$-actin (1:1,000; cat no. 60008-1-Ig). Subsequently, the PVDF membranes were washed with TBST three times (10 min each) and incubated with HRP-conjugated secondary goat anti-mouse $(1: 3,000$; cat no. SA00001-1) and goat anti-rabbit antibody (1:3,000; cat no. SA00001-2; all from ProteinTech Group, Inc.) for $2 \mathrm{~h}$ at $37^{\circ} \mathrm{C}$. The membranes were washed three times (10 min each) with TBST and protein bands were visualized using high sensitivity ECL regent (Boster Biological Technology) in an enhanced chemiluminescence (ChemiDoc) system (Bio-Rad Laboratories, Inc.) with hypersensitive ECL reagent (cat no. AR1170; Wuhan Boster Biological Technology, Ltd.).

Immunofluorescence staining. Total of $1 \times 10^{5} \mathrm{si}-\mathrm{NC}-$ and si-CLEC3A-transfected MG63 OS cells were fixed with 4\% paraformaldehyde for $10 \mathrm{~min}$ at room temperature, permeabilized with $0.5 \%$ Triton X-100 (Wuhan Servicebio Technology Co., Ltd.) for $10 \mathrm{~min}$ at room temperature and blocked with 5\% BSA (Wuhan Servicebio Technology Co., Ltd.) for $30 \mathrm{~min}$ at room temperature. The cells were subsequently incubated with rabbit anti-HIF1 $\alpha$ primary antibody $(1: 100$; cat no. 20960-1-AP; ProteinTech Group, Inc.) overnight at $4^{\circ} \mathrm{C}$. Following washing with PBS three times, MG63 cells were incubated with Cy3-conjugated anti-rabbit IgG for $2 \mathrm{~h}$ at room temperature. Nuclei were counterstained with DAPI for $10 \mathrm{~min}$ at room temperature and stained slides were visualized by fluorescence microscopy (magnification, x200).

Statistical analysis. Statistical analysis of three independent experimental repeats was performed using GraphPad Prism version 6.0 software (GraphPad Software, Inc.). Data were presented as mean \pm standard deviation. Significant differences between $>2$ groups was determined using one-way ANOVA and Fisher's Least Significant Difference post hoc test, whereas Student's t-tests were used to analyze the statistical significance between two groups. Co-expression relationships were analyzed using Pearson correlation analysis, with $\mathrm{R}>0.3$ and $\mathrm{P}<0.05$ set as cut-offs. Associations between CLEC3A expression and clinical characteristics were analyzed using $\chi^{2}$ tests, and patients were divided into high or low groups based the median expression value of CLEC3A. $\mathrm{P}<0.05$ was considered to indicate a statistically significant difference.

\section{Results}

CLEC3A expression is increased in OS tissue, and associated with TNM stage and lymph node metastasis. The gene expression profile data of GSE99671, including 18 tumor tissues and 18 adjacent normal bone tissues, were analyzed. Within the GSE99671 dataset, CLEC3A expression levels were found to be 

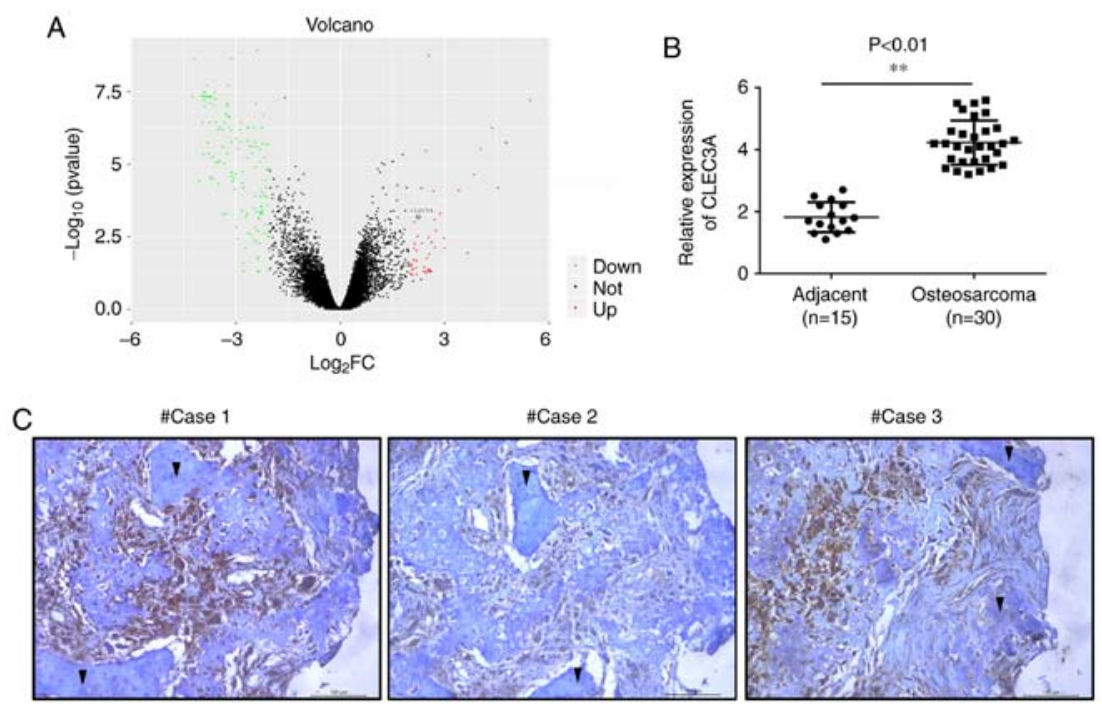

Figure 1. CLEC3A is highly expressed in OS tissues. (A) Volcano plot demonstrating the differently expressed genes in OS using the Gene Expression Omnibus data profile GSE99671 and CLEC3A is one of the upregulated gene (B) Reverse transcription-quantitative PCR was used to detect CLEC3A mRNA expression levels in clinical normal adjacent bone tissue $(\mathrm{n}=15)$ and OS tissue $(\mathrm{n}=30)$. (C) Representative micrographs demonstrating CLEC3A expression levels in OS and normal tissues. Black triangles indicate normal bone tissue infiltrated by OS cells. Scale bars, $100 \mu \mathrm{m} .{ }^{* *} \mathrm{P}<0.01$. OS, osteosarcoma; CLEC3A, C-type lectin domain family 3 member A.

A

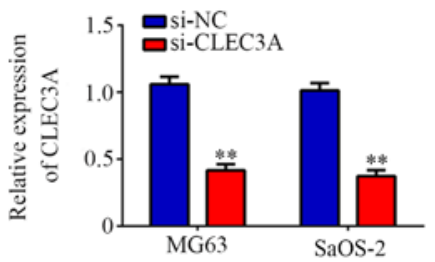

B
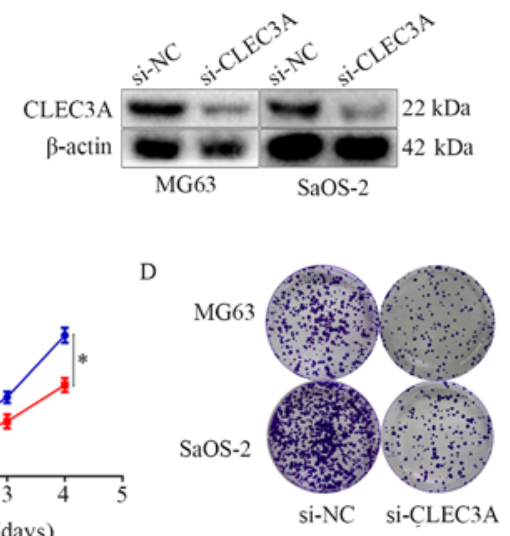

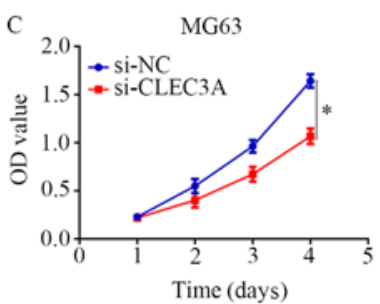

Time (days)
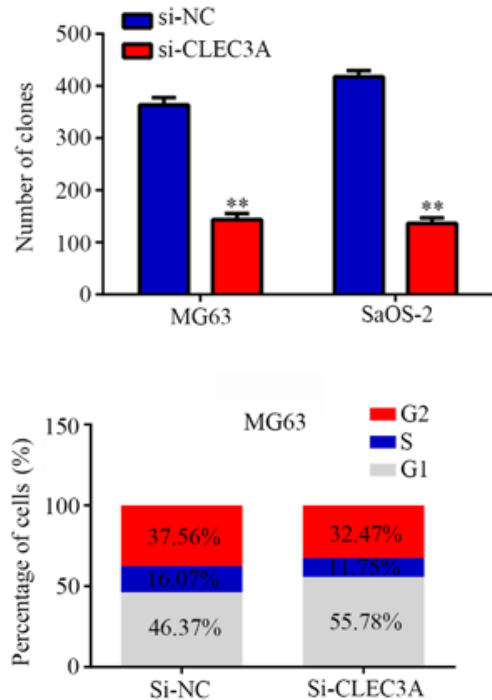

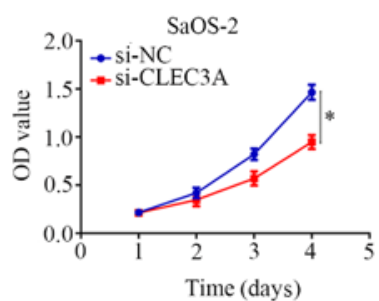

E si-NC
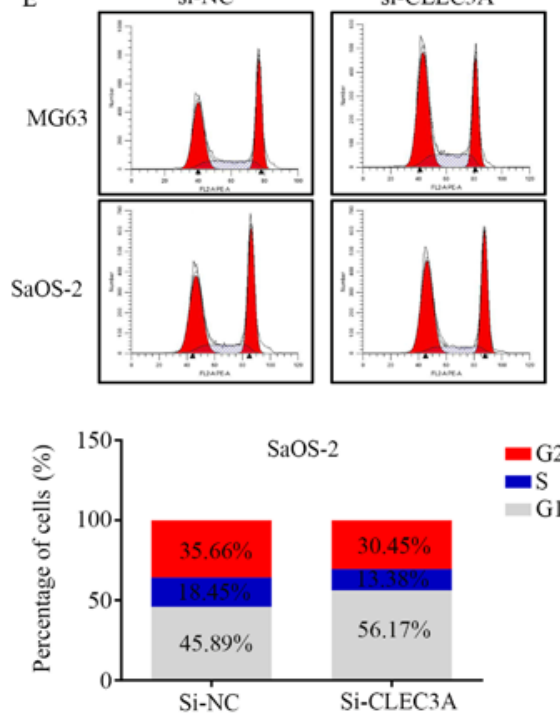

Figure 2. Genetic knockdown of CLEC3A with si-CLEC3A decreases the proliferation of OS cells and induces G1 phase arrest. Transfection efficiency of CLEC3A knockdown with si-CLEC3A compared with si-NC in MG63 and SaOS-2 cells detected using (A) reverse transcription-quantitative PCR and (B) western blotting. (C) Cell Counting Kit-8 assays were performed to detect the effect of CLEC3A knockdown with si-CLEC3A on OS cell proliferation. (D) Colony formation assays were performed to examine the effect of CLEC3A knockdown with si-CLEC3A on OS cell colony formation in MG63 and SaOS-2 cells (diameter of dishes $=6 \mathrm{~cm}$ ). (E) Cell cycle distribution was analyzed in the si-NC and si-CLEC3A groups in MG63 and SaOS-2 cells to assess the effect of CLEC3A on the cell cycle. ${ }^{*} \mathrm{P}<0.05$, ${ }^{* *} \mathrm{P}<0.01$. OS, osteosarcoma; CLEC3A, C-type lectin domain family 3 member A; si, small interfering RNA; NC, negative control; OD, optical density. 
Table I. Clinicopathological variables associated with 30 patients with osteosarcoma.

\begin{tabular}{|c|c|c|c|c|}
\hline \multirow[b]{2}{*}{ Variable } & \multirow[b]{2}{*}{$\mathrm{n}$} & \multicolumn{2}{|c|}{$\begin{array}{c}\text { C-type lectin domain } \\
\text { family } 3 \text { member A } \\
\text { expression }\end{array}$} & \multirow[b]{2}{*}{ P-value } \\
\hline & & High & Low & \\
\hline Age & & & & 0.713 \\
\hline$<60$ & 17 & 8 & 9 & \\
\hline$\geq 60$ & 13 & 7 & 6 & \\
\hline \multicolumn{5}{|c|}{ Tumor size $(\mathrm{cm})$} \\
\hline$<5$ & 16 & 10 & 6 & 0.143 \\
\hline$\geq 5$ & 14 & 5 & 9 & \\
\hline \multicolumn{5}{|c|}{ TNM stage } \\
\hline I-II & 14 & 4 & 10 & 0.028 \\
\hline III-IV & 16 & 11 & 5 & \\
\hline \multicolumn{5}{|c|}{ Lymph node metastasis } \\
\hline No & 12 & 3 & 9 & 0.025 \\
\hline Yes & 18 & 12 & 6 & \\
\hline
\end{tabular}

upregulated in OS tumor tissue compared with adjacent normal bone tissue (Fig. 1A). To verify these results, the expression levels of CLEC3A were analyzed in OS tissues from patients using RT-qPCR. The mRNA expression levels of CLEC3A were significantly increased in OS tissues $(n=30)$ compared with adjacent non-tumor tissues $(n=15 ;$ Fig. 1B). Subsequently, IHC staining was used to detect the protein expression levels of CLEC3A in OS tissues and observe the erosion of normal bone tissue by tumor cells; the protein expression levels of CLEC3A in OS cells were markedly high, whereas expression levels in normal bone cells were low (Fig. 1C). According to the mRNA expression level of CLEC3A, patient OS tissues were divided into low expression and high expression groups (Table I), and the expression of CLEC3A was demonstrated to be positively associated with TNM stage and lymph node metastasis, but not age or tumor size (Table I).

Inhibition of CLEC3A decreases OS cell proliferation and induces G1 phase arrest. To investigate the role of CLEC3A in OS, si-CLEC3A was used to transiently knock down CLEC3A in the OS cell lines MG63 and SaOS-2; the transfection efficiency was confirmed as successful following RT-qPCR and western blotting analyses of mRNA and protein expression levels in cells transfected with si-CLEC3A compared with si-NC (Fig. 2A and B). The silencing of CLEC3A with si-CLEC3A significantly decreased the proliferation of MG63 and SaOS-2 OS cells at day 4 compared with si-NC-transfected cells (Fig. 2C). Colony formation assays were subsequently performed to evaluate the effects of CLEC3A on OS cell colony forming ability; the results demonstrated that knockdown of CLEC3A significantly decreased the number of colonies formed by both MG63 and SaOS-2 cells compared with si-NC-transfected cells (Fig. 2D). Furthermore, si-CLEC3A-transfected cells were observed to have an increased proportion of cells in the G1 phase compared with the NC (Fig. 2E). Taken together, these
Table II. $\mathrm{IC}_{50}$ values of doxorubicin and cisplatin in si-NC and si-CLEC3A knockdown MG63 and SaOS-2 cells at 24, 48 and $72 \mathrm{~h}$ (mean \pm standard deviation).

A, Doxorubicin

\begin{tabular}{llll}
\hline & \multicolumn{2}{c}{$\mathrm{IC}_{50}(\mu \mathrm{M})$} & \\
\cline { 2 - 3 } Group & $24 \mathrm{~h}$ & $48 \mathrm{~h}$ & $72 \mathrm{~h}$ \\
\hline MG63-si-NC & $2.66 \pm 0.13$ & $1.89 \pm 0.11$ & $1.65 \pm 0.07$ \\
MG63-si-CLEC3A & $0.87 \pm 0.15^{\mathrm{a}}$ & $0.34 \pm 0.05^{\mathrm{a}}$ & $0.28 \pm 0.07^{\mathrm{a}}$ \\
SaOS-2-si-NC & $3.01 \pm 0.17$ & $2.03 \pm 0.13$ & $1.77 \pm 0.06$ \\
SaOS-2-si-CLEC3A & $1.39 \pm 0.11^{\mathrm{a}}$ & $0.51 \pm 0.09^{\mathrm{a}}$ & $0.43 \pm 0.05^{\mathrm{a}}$ \\
\hline
\end{tabular}

B, Cisplatin

\begin{tabular}{|c|c|c|c|}
\hline \multirow[b]{2}{*}{ Group } & \multicolumn{2}{|c|}{$\mathrm{IC}_{50}(\mu \mathrm{M})$} & \multirow[b]{2}{*}{$72 \mathrm{~h}$} \\
\hline & $24 \mathrm{~h}$ & $48 \mathrm{~h}$ & \\
\hline MG63-si-NC & $2.33 \pm 0.14$ & $1.54 \pm 0.06$ & $1.24 \pm 0.09$ \\
\hline MG63-si-CLEC3A & $0.67 \pm 0.13^{\mathrm{a}}$ & $0.29 \pm 0.06^{\mathrm{a}}$ & $0.19 \pm 0.03$ \\
\hline SaOS-2-si-NC & $2.77 \pm 0.21$ & $1.77 \pm 0.11$ & $1.45 \pm 0.04$ \\
\hline SaOS-2-si-CLEC3A & $1.23 \pm 0.14^{\mathrm{a}}$ & $0.42 \pm 0.07^{\mathrm{a}}$ & $0.31 \pm 0.05^{\circ}$ \\
\hline
\end{tabular}

${ }^{\text {a }}<0.05$. si, small interfering RNA; CLEC3A, C-type lectin domain family 3 member A; NC, negative control.

findings suggested that the genetic knockdown of CLEC3A may decrease OS cell proliferation and induce G1 phase arrest.

Overexpression of CLEC3A increases cell proliferation and decreases 61 phase arrest. A CLEC3A overexpression plasmid was used to construct CLEC3A-overexpressing MG63 cells and SaOS-2 cells; the transfection efficiency was confirmed as successful following RT-qPCR and western blotting analyses of mRNA and protein expression levels in cells overexpressing CLEC3A compared with NC cells (Fig. 3A and B). CCK-8 assays revealed that the overexpression of CLEC3A significantly increased the proliferation rate at 4 days of MG63 and SaOS-2 cells compared with the NC (Fig. 3C). Similarly, through colony formation assays, it was observed that the overexpression of CLEC3A significantly increased the number of colonies formed in both MG63 and SaOS-2 cells compared with the NC (Fig. 3D). Furthermore, cell cycle distribution analysis found that the proportion of cells in G1 phase was decreased in the CLEC3A overexpression group, whereas the proportion of cells increased in the $S$ phase compared with the NC group (Fig. 3E).

CLEC3A knockdown promotes chemosensitivity of OS cells to doxorubicin and cisplatin. Chemotherapy resistance is an important reason for the failure of OS treatment (12). To determine whether the genetic knockdown of CLEC3A can promote chemosensitivity in OS cells, MG63 and SaOS-2 cells were treated with doxorubicin (DOX) and cisplatin (CDDP) for 24, 48 and $72 \mathrm{~h}$. The $\mathrm{IC}_{50}$ values of DOX and CDDP for MG63 
A

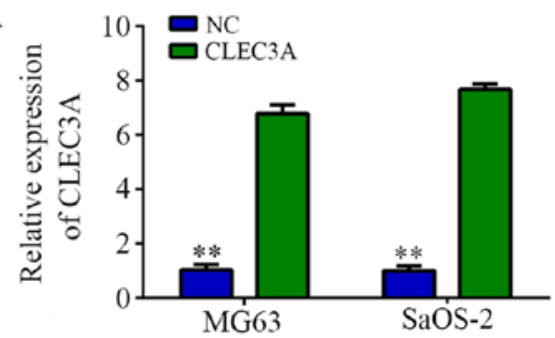

B

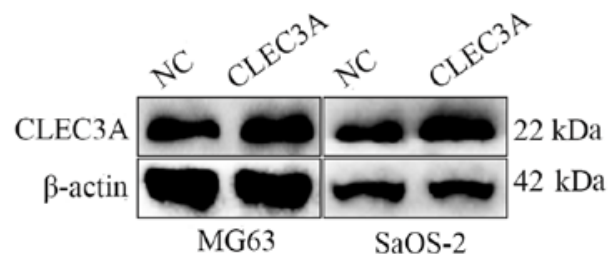

$\mathrm{C}$
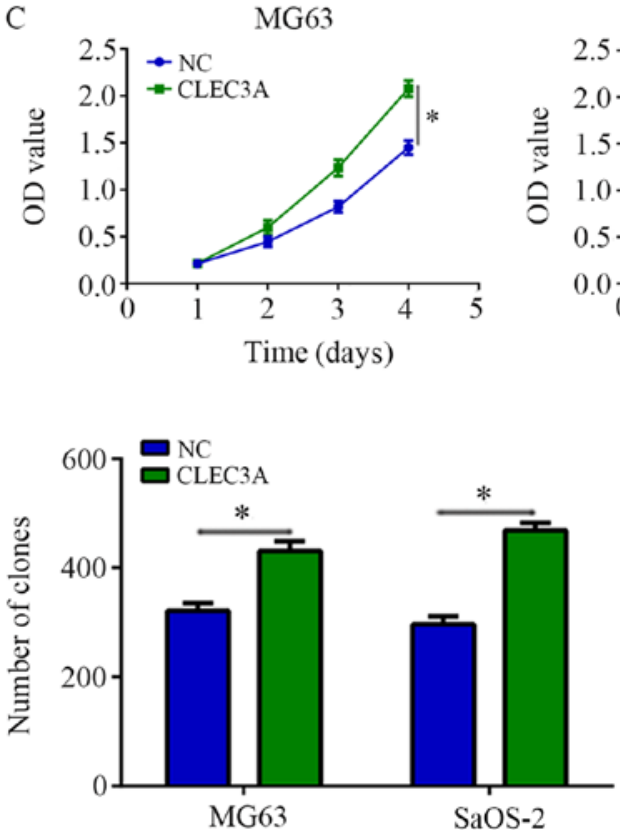

$\mathrm{SaOS}-2$

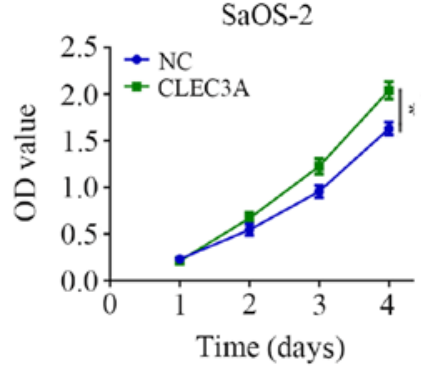

E

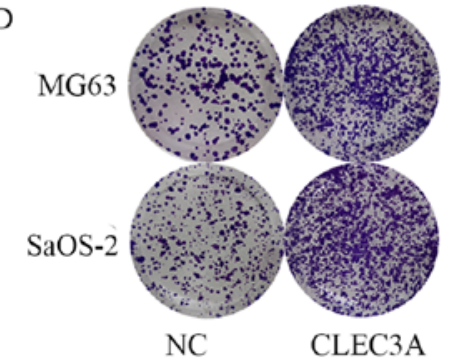

CLEC3A NC CLEC3A

SaOS-2
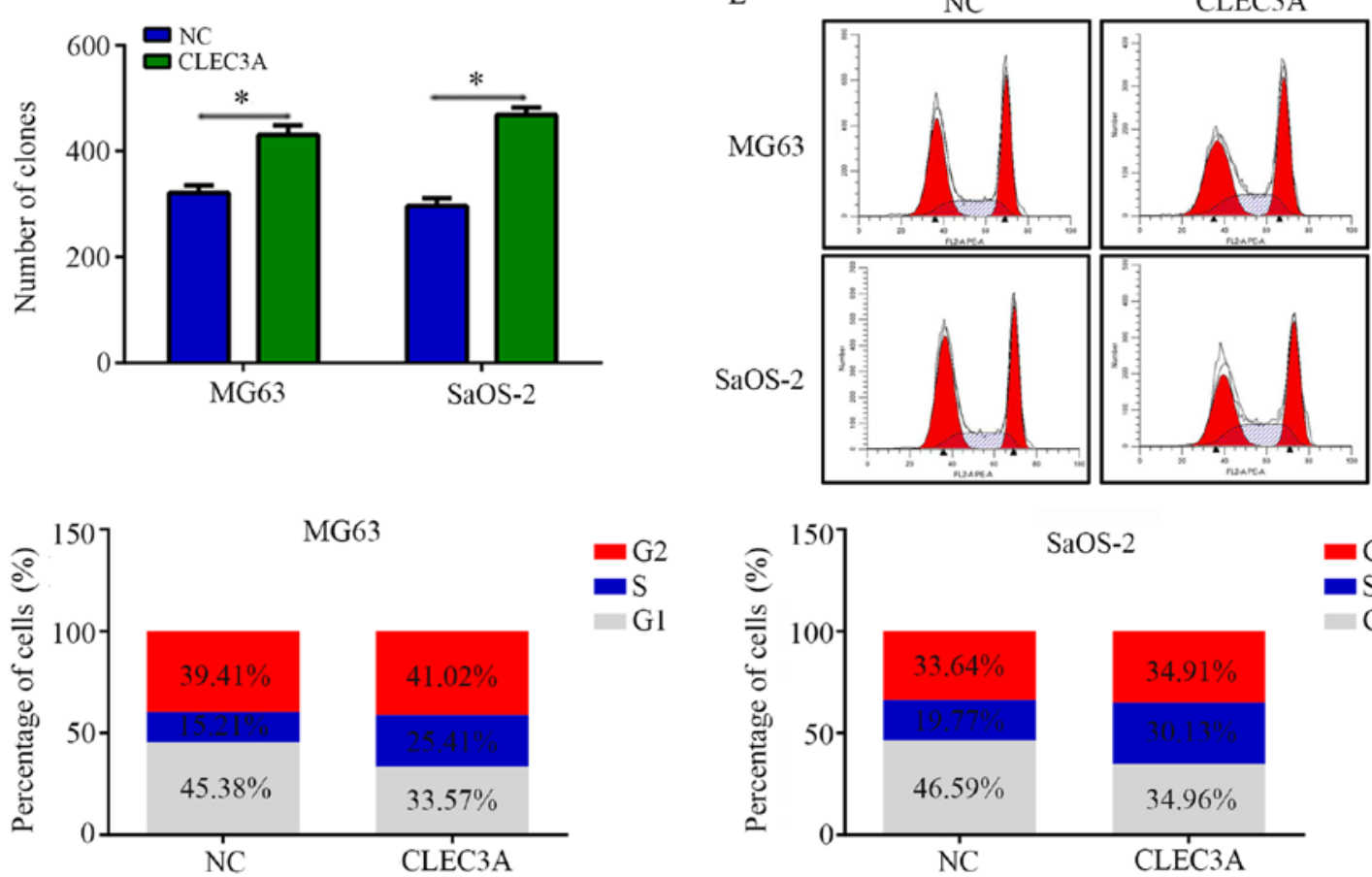

Figure 3. Overexpression of CLEC3A increases the proliferation of OS cells and decreases G1 phase arrest. Transfection efficiency of CLEC3A overexpression plasmid compared empty plasmid (NC) in MG63 and SaOS-2 cells detected using (A) reverse transcription-quantitative PCR and (B) western blotting. (C) Cell Counting Kit-8 assays were performed to detect the effect of CLEC3A overexpression on OS cell proliferation. (D) Colony formation assays were performed to examine the effect of CLEC3A overexpression on OS cell colony formation in MG63 and SaOS-2 cells (diameter of dishes=6 cm). (E) Cell cycle distribution was analyzed in the NC group and CLEC3A-overexpressing group in MG63 and SaOS-2 cells to assess the effect of CLEC3A on the cell cycle. "P $<0.05$, ${ }^{* *} \mathrm{P}<0.01$. OS, osteosarcoma; CLEC3A, C-type lectin domain family 3 member A; NC, negative control; OD, optical density.

and SaOS-2 cells at 24, 48 and $72 \mathrm{~h}$ are presented in Table II. Furthermore, DOX and CDDP significantly decreased colony formation compared with normal control, while CLEC3A inhibition combined with DOX or CDDP treatment significantly decreased the colony number compared with DOX or CDDP treatment alone (Fig. 4A and B). These results indicated that the knockdown of CLEC3A may promote chemosensitivity of MG63 and SaOS-2 OS cells to DOX and CDDP.

CLEC3A regulates the AKT1/mTOR/HIFl $\alpha$ signaling pathway. Various studies have reported that the AKT1/mTOR pathway is involved in cancer cell proliferation, migration, metastasis and drug susceptibility $(13,14)$. Through analyzing the relationship between CLEC3A and AKT1 in the 30 OS patient tissues, it was observed that CLEC3A was positively correlated with AKT1 (Fig. 5A). Therefore, it was hypothesized that CLEC3A may have the potential to regulate the AKT1/mTOR pathway. Western blotting was performed, and the results found that CLEC3A knockdown decreased the expression of AKT1 and mTOR in MG63 and SaOS-2 cells compared with si-NC-transfected cells (Fig. 5B). HIF1 $\alpha$ is a critical protein regulated by AKT1/mTOR; increased levels of HIF1 $\alpha$ translocate to the 

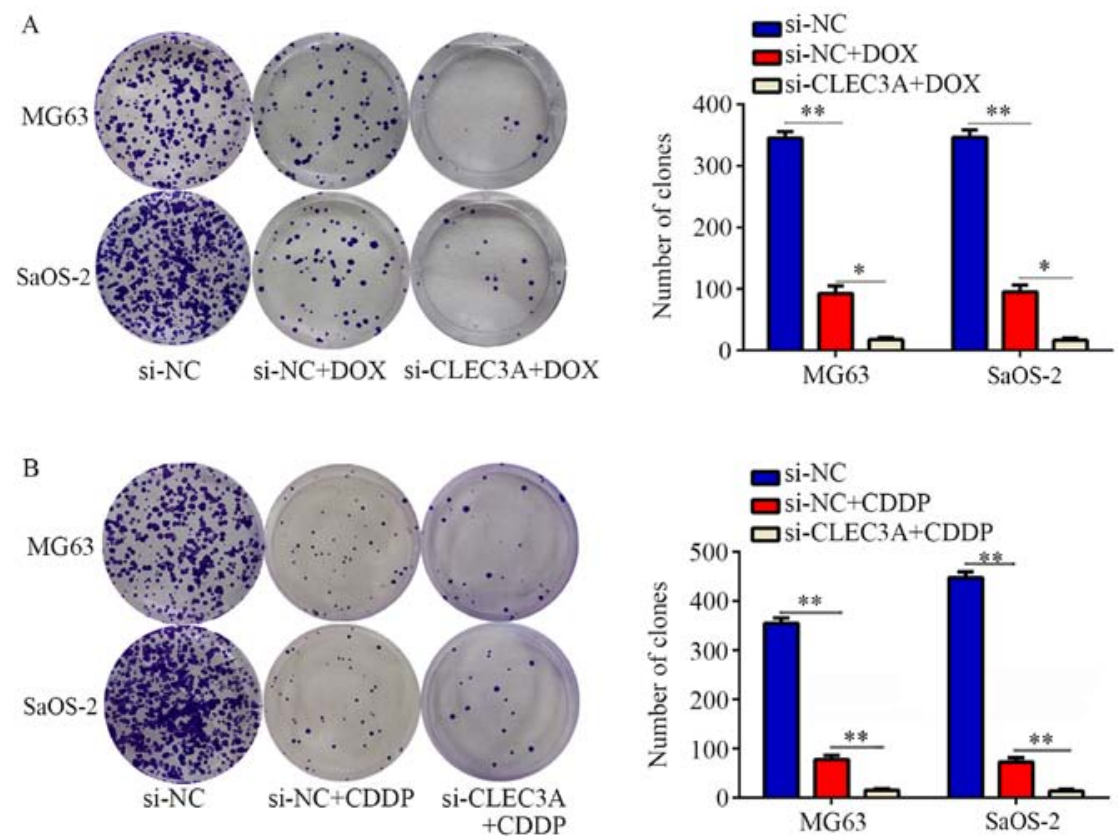

Figure 4. CLEC3A suppression increases the chemosensitivity of osteosarcoma cells to DOX and CDDP. Colony formation assays were performed to detect the effect of (A) DOX $(2 \mu \mathrm{M})$ or (B) CDDP $(2 \mu \mathrm{M})$ in si-NC-transfected cells or si-CLEC3A-transfected cells (diameter of dishes $=6 \mathrm{~cm}) .{ }^{*} \mathrm{P}<0.05$, ${ }^{* *} \mathrm{P}<0.01$. CLEC3A, C-type lectin domain family 3 member A; NC, negative control; DOX, doxorubicin; CDDP, cisplatin; si, small interfering RNA.
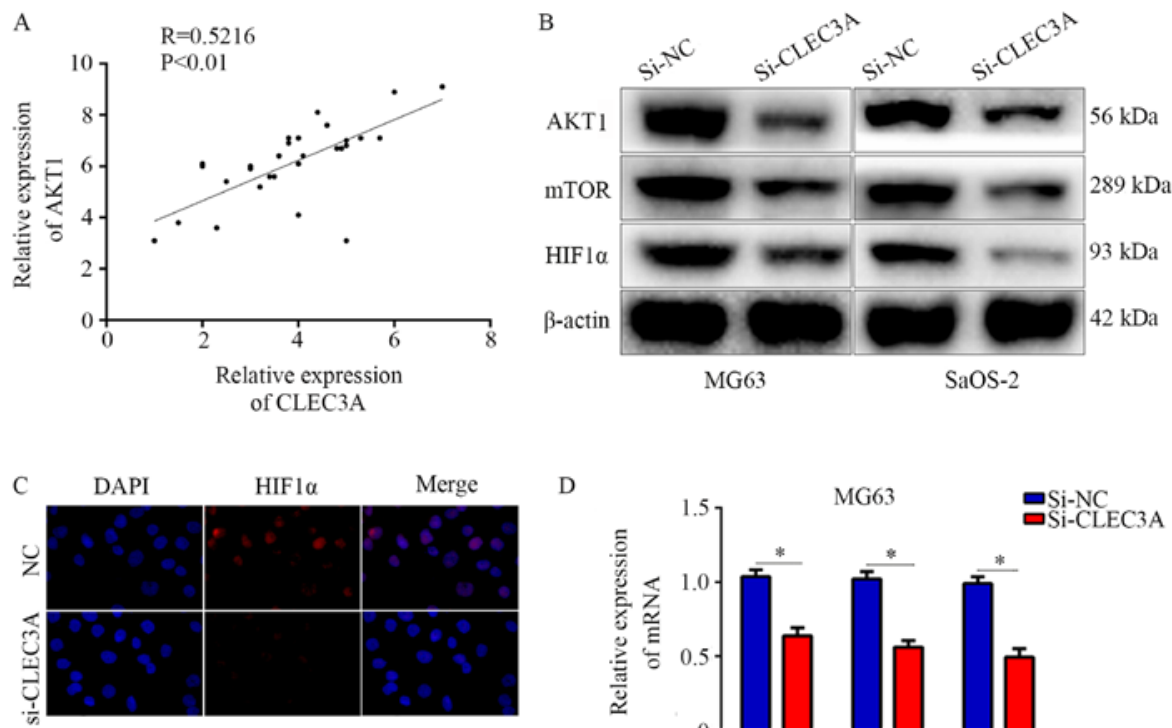

D
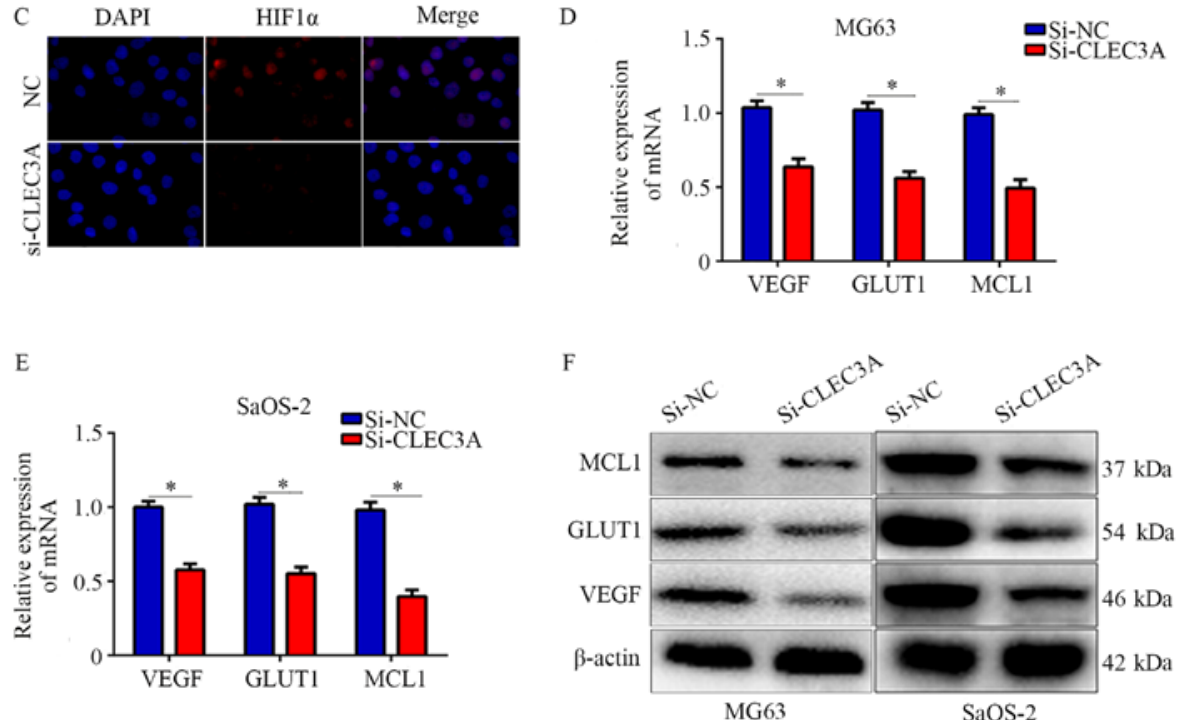

Figure 5. Knockdown of CLEC3A suppresses the AKT1/mTOR/HIF1 $\alpha$ pathway. (A) Scatter diagram demonstrating the co-expressing relationship between CLEC3A and AKT1 in mRNA level in patient OS tissues. (B) Expression levels of AKT1, mTOR, and HIF1 $\alpha$ in the si-NC- and si-CLEC3A-transfected MG63 and SaOS-2 cells was detected using western blotting. (C) Immunofluorescence was used to analyze the nuclear location of HIFl $\alpha$ in the si-NC- and si-CLEC3A-transfected MG63 cells (magnification, x200). VEGF, GLUT1 and MCL1 mRNA expression levels were detected by reverse transcription-quantitative PCR in the si-NC- and si-CLEC3A-transfected (D) MG62 and (E) SaOS-2 cells. (F) VEGF, GLUT1, and MCL1 protein expression levels were detected in the si-NC- and si-CLEC3A-transfected cells by western blotting."P<0.05. OS, osteosarcoma; CLEC3A, C-type lectin domain family 3 member A; NC, negative control; si, small interfering RNA; HIF1', hypoxia-inducible factor-1'; VEGF, vascular endothelial growth factor; GLUT1, glucose transporter 1; MCL1, induced myeloid leukemia cell differentiation protein Mcl-1. 

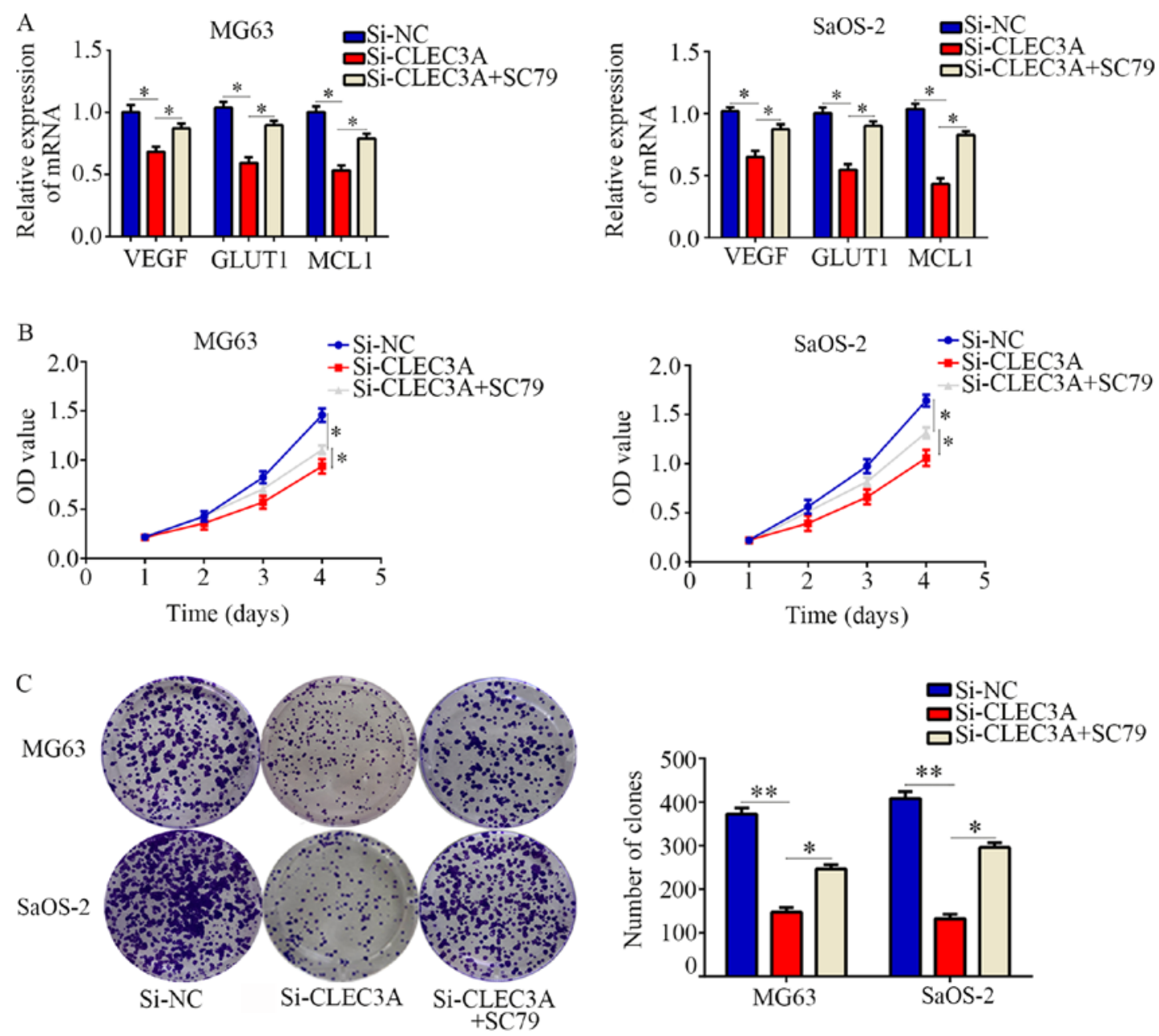

Figure 6. Restored AKT1/mTOR/HIF1 $\alpha$ pathway expression reverses the effect of CLEC3A knockdown on biological functions. (A) Reverse transcription-quantitative PCR was used to detect the mRNA expression levels of VEGF, GLUT1 and MCL1 in si-NC- and si-CLEC3A-transfected MG63 and SaOS-2 cells with or without treatment with SC79. (B) Cell Counting Kit-8 assays were used to detect the rate of cell proliferation in si-NC- and si-CLEC3A-transfected MG63 and SaOS-2 cells with or without treatment with SC79. (C) Colony formation assays were used to detect the colony forming ability in si-NC- and si-CLEC3A-transfected cells with or without treatment with SC79 (diameter of dishes $=6 \mathrm{~cm}$ ). ${ }^{*} \mathrm{P}<0.05$, ${ }^{* *} \mathrm{P}<0.01$. CLEC3A, C-type lectin domain family 3 member A; NC, negative control; si, small interfering RNA; VEGF, vascular endothelial growth factor; GLUT1, glucose transporter 1; MCL1, myeloid cell leukemia 1.

nucleus to promote various cancer cell processes, such as proliferation and migration (15). Therefore, the expression of HIF1 $\alpha$ was investigated; the expression levels of HIF1 $\alpha$ were also decreased when CLEC3A was knocked down compared to the NC group (Fig. 5B). Immunofluorescence was also used to detect the intracellular localization of HIF1 $\alpha$ in the $\mathrm{NC}$ group and si-CLEC3A group. It was observed that HIF1 $\alpha$ was mainly located in the nucleus in the NC group under normal culture conditions, whereas the genetic knockdown of CLEC3A reduced the amount of HIF1 $\alpha$ accumulated in the nucleus (Fig. 5C). Moreover, the mRNA and protein expression levels of HIF1 $\alpha$-target genes, including VEGF, GLUT1 and MCL1 were decreased following CLEC3A knockdown in MG63 and SaOS-2 cells compared with si-NC-transfected cells (Fig. 5D-F). These data indicated that CLEC3A may regulate the AKT1/mTOR/HIF1 $\alpha$ pathway.

Restoration of AKT activity reverses the effect of CLEC3A on $O S$ cell proliferation. To determine whether the AKT1/mTOR/HIF1 $\alpha$ pathway is involved in CLEC3A-induced biological functions, an AKT activator, SC79, was employed.
CLEC3A inhibition significantly decreased the expression of HIF1 $\alpha$ target genes compared with normal control cells, and then SC79 restored them (Fig. 6A). CCK-8 assays demonstrated that SC79-activated AKT also significantly reversed the inhibitory effects of $C L E C 3 A$ knockdown on OS cell proliferation (Fig. 6B). In addition, it was observed that SC79-activated AKT could significantly reverse the effect of CLEC3A knockdown on OS cell colony forming ability (Fig. 6C). Taken together, these results indicated that CLEC3A may promote OS cell proliferation via the AKT1/mTOR/HIF1 $\alpha$ signaling pathway.

\section{Discussion}

OS is a malignant tumor that occurs in bone tissues and mainly affects adolescents (16). Through developments in diagnostic and therapeutic techniques, the 5-year survival rate of patients with OS has increased by $60-70 \%$ in the last decade; however, the 20-year survival rate of patients with OS remains low, at $20 \%(17,18)$. The mechanisms involved in the development of OS are largely unclear; thus, the identification 
of novel biomarkers in OS may be useful for future diagnosis and treatment of patients.

Dysregulated C-type lectins have been found in various diseases, including cancer (19,20). CLEC-2, a member of the C-type lectin family, was highly expressed in clear cell renal cell carcinoma and was positively associated with poor patient prognosis (21) and it was also observed to regulate cell proliferation and migration (22). CLEC3A, another C-type lectin, was reported to be highly expressed in breast invasive ductal carcinoma and promoted breast cancer cell proliferation and migration (8). A previous study has demonstrated that CLEC3A is involved in bone formation (23); however, the effect of CLEC3A on OS cells is largely unknown. In the present study, consistent with its role in breast cancer (8), it was demonstrated that CLEC3A is highly expressed in OS tissues, and this high expression of CLEC3A was positively associated with TNM stage and lymph node metastasis. These were the first evidences which demonstrated that CLECE3A may be a novel biomarker for OS. Furthermore, biology function experiments showed CLEC3A knockdown decreased OS cell proliferation, and increased the chemosensitivity of OS cells to DOX and CDDP, whereas the overexpression of CLEC3A increased cell proliferation. These were first evidences which demonstrated that CELEC3A may be an oncogene in OS.

Numerous studies have demonstrated that the change in expression levels of genes involved in the $\mathrm{PI}$ KK/AKT/mTOR pathway are common in osteosarcoma $(24,25)$. The PI3K/AKT/mTOR pathway promoted the development of osteosarcoma through regulating a series of target genes, such as mouse double minute 2 homolog/p53, cyclins and matrix metalloproteinases (26). Similarly, various genes have demonstrated the potential to regulate the $\mathrm{PI} 3 \mathrm{~K} / \mathrm{AK} T / \mathrm{mTOR}$ pathway $(27,28)$. In the present study, it was found that the knockdown of CLEC3A decreased the expression levels of AKT/mTOR. HIF1 $\alpha$ is a critical transcriptional regulator of the adaptive response to hypoxia. Under normoxia, HIF1 $\alpha$ is modified by oxygen-dependent prolyl-hydroxylases and is subsequently recognized by the von Hippel-Lindau tumor suppressor protein and degraded by the ubiquitination pathway (29). However, under hypoxia, oxygen-dependent prolyl hydroxylases are inactive and HIF1 $\alpha$ is stable, facilitating its dimerization with HIF-1 $\beta$, translocation to the nucleus and binding to the promoters of target genes to promote their transcription $(30,31)$. The expression of HIF1 $\alpha$ and its activity are also controlled by oxygen-independent mechanisms; for example, multiple studies have reported that the AKT1/mTOR signaling pathway increased the expression of HIF1 $\alpha(32,33)$. In the present study, the genetic knockdown of CLEC3A significantly decreased both the protein expression levels of HIF1 $\alpha$ and its nuclear localization. Various studies have shown that the target genes of HIF1 $\alpha$ such as VEGF, MCL1 and Glut1 induced the metabolic change and apoptosis inhibition, therefore promoting proliferation and decreasing sensitivity of chemotherapy $(34,35)$. In the present study, we showed the expression levels of HIF1 $\alpha$-targeted genes, including MCL1, GLUT1 and VEGF, were also decreased when CLEC3A was knocked down; however, the restoration of the AKT1/mTOR/HIF1 $\alpha$ signaling with SC79 reversed the inhibitory effects of CLEC3A knockdown on the biological function of OS cells.
In conclusion, the present study suggested that CLEC3A may be an oncogene in OS by promoting OS cell proliferation and demonstrated that CLEC3A may be involved in chemosensitivity through regulating the AKT1/mTOR/HIF1 $\alpha$ signaling pathway. Thus, CLEC3A may contribute to the development of OS and be a potential target for OS diagnosis and treatment.

\section{Acknowledgements}

Not applicable.

\section{Funding}

This work was supported by Qian Kehe SY [grant no. (2013)3042] and The National Natural Science Foundation of China (grant no. 81560356).

\section{Availability of data and materials}

The datasets used and/or analyzed during the current study are available from the corresponding author on reasonable request.

\section{Authors' contributions}

CR, RP, LH, HW, JS and WZ were responsible for performing the experiments, collecting the data, conducting the data analysis and interpreting the results. XT and HC designed the experiments and wrote the manuscript. All authors read and approved the final version of the article.

\section{Ethics approval and consent to participate}

The present study was approved by the Ethics Committee of the Guiyang Maternal and Child Health-Care Hospital and was performed in accordance with the principles embodied in the Declaration of Helsinki. Informed consent was obtained from all patients who provided samples.

\section{Patient consent for publication}

Not applicable.

\section{Competing interests}

The authors declare that they have no competing interests.

\section{References}

1. Maximov VV, Akkawi R, Khawaled S, Salah Z, Jaber L, Barhoum A, Or O, Galasso M, Kurek KC, Yavin E and Aqeilan RI: MiR-16-1-3p and miR-16-2-3p possess strong tumor suppressive and antimetastatic properties in osteosarcoma. Int J Cancer 145: 3052-3063, 2019.

2. Hsu MJ, Peng SF, Chueh FS, Tsai CH, Tsai FJ, Huang CY, Tang CH, Yang JS, Hsu YM, Huang WW and Chung JG: Lupeol suppresses migration and invasion via p38/MAPK and PI3K/Akt signaling pathways in human osteosarcoma U-2 OS cells. Biosci Biotechnol Biochem 83: 1729-1739, 2019.

3. Ma H, Su R, Feng H, Guo Y and Su G: Long noncoding RNA UCA1 promotes osteosarcoma metastasis through CREB1-mediated epithelial-mesenchymal transition and activating PI3K/AKT/mTOR pathway. J Bone Oncol 16: 100228, 2019. 
4. Wang Y, Huang $\mathrm{H}$ and Li Y: Knocking down miR-384 promotes growth and metastasis of osteosarcoma MG63 cells by targeting SLBP. Artif Cells Nanomed Biotechnol 47: 1458-1465, 2019.

5. Chen JK, Peng SF, Lai KC, Liu HC, Huang YP, Lin CC, Huang AC, Chueh FS and Chung JG: Fistein suppresses human osteosarcoma U-2 OS cell migration and invasion via affecting FAK, $\mathrm{UPA}$ and NF- $\mathrm{KB}$ signaling pathway in vitro. In vivo 33: 801-810, 2019.

6. Lau D, Elezagic D, Hermes G, Mörgelin M, Wohl AP, Koch M, Hartmann U, Hölliriegl S, Wagener R, Paulsson M, et al: The cartilage-specific lectin C-type lectin domain family 3 member A (CLEC3A) enhances tissue plasminogen activator-mediated plasminogen activation. J Biol Chem 293: 203-214, 2018.

7. Wang Y, Lv Y, Liu TS, Yan WD, Chen LY, Li ZH, Piao YS, An RB, Lin ZH and Ren XS: Cordycepin suppresses cell proliferation and migration by targeting CLEC 2 in human gastric cancer cells via Akt signaling pathway. Life Sci 223: 110-119, 2019.

8. Ni J,Peng Y, Yang FL, Xi X, Huang XW and He C: Overexpression of CLEC3A promotes tumor progression and poor prognosis in breast invasive ductal cancer. Onco Targets Ther 11: 3303-3312, 2018.

9. Ho XD, Phung P, Q Le V, H Nguyen V, Reimann E, Prans E, Kõks G, Maasalu K, Le NT, H Trinh L, et al: Whole transcriptome analysis identifies differentially regulated networks between osteosarcoma and normal bone samples. Exp Biol Med (Maywood) 242: 1802-1811, 2017.

10. Sepulveda JL: Using $\mathrm{R}$ and Bioconductor in clinical genomics and transcriptomics. J Mol Diagn 22: 3-20, 2019.

11. Livak JK and Schmittgen TD: Analysis of relative gene expression data using quantitative PCR and the 2 (-Delta Delta C(T)) method. Methods 25: 402-408, 2001.

12. Heng M, Gupta A, Chung PW, Healey JH, Vaynrub M, Rose PS, Houdek MT, Lin PP, Bishop AJ, Hornicek FJ, et al: The role of chemotherapy and radiotherapy in localized extraskeletal osteosarcoma. Eur J Cancer 125: 130-141, 2010.

13. Pan C, Liu Q and Wu X: HIF1 $\alpha$ a/miR-520a-3p/AKT1/mTOR feedback promotes the proliferation and glycolysis of gastric cancer cells. Cancer Manag Res 11: 10145-10156, 2019.

14. Zhang X, Wang S, Wang H, Cao J, Huang X, Chen Z, Xu P, Sun G, Xu J, Lv J and Xu Z: Circular RNA circNRIP1 acts as a microRNA-149-5p sponge to promote gastric cancer progression via the AKT1/mTOR pathway. Mol Cancer 18: 20, 2019.

15. Liao Z, She C, Ma L, Sun Z, Li P, Zhang X, Wang P and Li W: KDELR2 promotes glioblastoma tumorigenesis targeted by HIF1a via mTOR signaling pathway. Cell Mol Neurobiol 39: 1207-1215, 2019.

16. Prater S and McKeon B: Cancer, Osteosarcoma. StatPearls Publishing, Treasure Island, FL, 2019.

17. Imura $Y$, Takenaka $S$, Kakunaga S, Nakai T, Wakamatsu $T$, Outani H, Tanaka T, Tamiya H, Oshima K, Hamada K, et al: Survival analysis of elderly patients with osteosarcoma. Int Orthop 43: 1741-1747, 2019.

18. Zhang Y, Yang J, Zhao N, Wang C, Kamar S, Zhou Y, He Z, Yang J, Sun B, Shi X, et al: Progress in the chemotherapeutic treatment of osteosarcoma. Oncol Lett 16: 6228-6237, 2018.

19. Mayer S, Raulf MK and Lepenies B: C-type lectins: Their network and roles in pathogen recognition and immunity. Histochem Cell Biol 147: 223-237, 2017.

20. Ding D, Yao Y, Zhang S, Su C and Zhang Y: C-type lectins facilitate tumor metastasis. Oncol Lett 13: 13-21, 2017.

21. Xiong Y, Liu L, Xia Y, Wang J, Xi W, Bai Q, Qu Y, Long Q, Xu J and Guo J: High CLEC-2 expression associates with unfavorable postoperative prognosis of patients with clear cell renal cell carcinoma. Oncotarget 7: 63661-63668, 2016.
22. Osada M, Inoue O, Ding G, Shirai T, Ichise H, Hirayama K, Takano K, Yatomi Y, Hirashima M, Fujii H, et al: Platelet activation receptor CLEC-2 regulates blood/lymphatic vessel separation by inhibiting proliferation, migration, and tube formation of lymphatic endothelial cells. J Biol Chem 287: 22241-22252, 2012.

23. Karlsson C, Dehne T, Lindahl A, Brittberg M, Pruss A, Sittinger $\mathrm{M}$ and Ringe J: Genome-wide expression profiling reveals new candidate genes associated with osteoarthritis. Osteoarthritis Cartilage 18: 581-592, 2010.

24. Perry JA, Kiezun A, Tonzi P, Van Allen EM, Carter SL, Baca SC, Cowley GS, Bhatt AS, Rheinbay E, Pedamallu CS, et al: Complementary genomic approaches highlight the PI3K/mTOR pathway as a common vulnerability in osteosarcoma. Proc Natl Acad Sci USA 111: E5564-E5573, 2014

25. Zhang J, Yu XH, Yan YG, Wang C and Wang WJ: PI3K/Akt signaling in osteosarcoma. Clin Chim Acta 444: 182-192, 2015.

26. Hu K, Dai HB and Qiu ZL: mTOR signaling in osteosarcoma: Oncogenesis and therapeutic aspects (Review). Oncol Rep 36: 1219-1225, 2016.

27. Jiang C, Ma Z, Zhang G, Yang X, Du Q and Wang W: CSNK2A1 Promotes gastric cancer invasion through the PI3K-Akt-mTOR signaling pathway. Cancer Manag Res 11: 10135-10143, 2019.

28. Li ZQ, Qu M, Wan HX, Wang H, Deng Q and Zhang Y: FOXK1 promotes malignant progression of breast cancer by activating PI3K/AKT/mTOR signaling pathway. Eur Rev Med Pharmacol Sci 23: 9978-9987, 2019.

29. Qian J and Rankin EB: Hypoxia-induced phenotypes that mediate tumor heterogeneity. Adv Exp Med Biol 1136: 43-55, 2019.

30. Hayashi Y, Yokota A, Harada H and Huang G: Hypoxia/pseudohypoxia-mediated activation of hypoxia-inducible factor- $1 \alpha$ in cancer. Cancer Sci 110: 1510-1517, 2019.

31. Thomas LW and Ashcroft M: Exploring the molecular interface between hypoxia-inducible factor signalling and mitochondria. Cell Mol Life Sci 76: 1759-1777, 2019.

32. Du Y, Long Q, Zhang L, Shi Y, Liu X, Li X, Guan B, Tian Y, Wang X, Li L and He D: Curcumin inhibits cancer-associated fibroblast-driven prostate cancer invasion through MAOA/mTOR/HIF-1 $\alpha$ signaling. Int J Oncol 47: 2064-2072, 2015.

33. Woo YM, Shin Y, Lee EJ, Lee S, Jeong SH, Kong HK, Park EY, Kim HK, Han J, Chang M and Park JH: Inhibition of aerobic glycolysis represses Akt/mTOR/HIF- $1 \alpha$ axis and restores tamoxifen sensitivity in antiestrogen-resistant breast cancer cells. PLoS One 10: e0132285, 2015.

34. Wang Y, Huang Y,Liu H,Su D, Luo F and Zhou F: Long noncoding RNA CDKN2B-AS1 interacts with miR-411-3p to regulate ovarian cancer in vitro and In vivo through HIF-1a/VEGF/P38 pathway. Biochem Biophys Res Commun 514: 44-50, 2019.

35. Sadlecki P, Bodnar M, Grabiec M, Marszalek A, Walentowicz P, Sokup A, Zegarska J and Walentowicz-Sadlecka M: The role of Hypoxia-inducible factor-1 $\alpha$, glucose transporter-1, (GLUT-1) and carbon anhydrase IX in endometrial cancer patients. BioMed research international 2014: 616850, 2014.

This work is licensed under a Creative Commons Attribution-NonCommercial-NoDerivatives 4.0 International (CC BY-NC-ND 4.0) License. 